\title{
Burnout y calidad de vida en traumatólogos chilenos: ¿Estamos realmente bien?
}

\section{Burnout and Quality of Life in Chilean Orthopaedic Surgeons: Are we doing ok?}

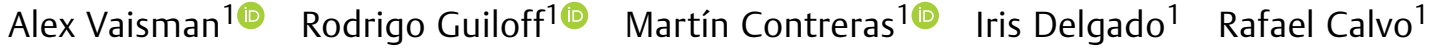 \\ Francisco Figueroa $^{1}$ David Figueroa ${ }^{1}$ \\ ${ }^{1}$ Facultad de Medicina Clínica Alemana, Universidad del Desarrollo, \\ Santiago, Chile \\ Address for correspondence Rodrigo Guiloff, MD, Clinica Alemana de \\ Santiago SA, Av. Vitacura 5951 Santiago, Chile \\ (e-mail: rguiloff@gmail.com).
}

Rev Chil Ortop Traumatol 2020;61:76-82.

\begin{abstract}
Resumen
Introducción No existen reportes sobre el índice de burnout en traumatólogos que ejercen en Chile.

Objetivo Evaluar la presencia de burnout y otros aspectos de calidad de vida en esa población.

Material y Método Estudio transversal analítico. Encuesta diseñada para conocer el auto-reporte de burnout y síntomas depresivos entre otros factores. Se incluyeron variables demográficas, laborales, sociales, hábitos personales y anímicas. La encuesta fue compartida vía online a miembros de la Sociedad Chilena de Ortopedia y Traumatología. Se realizó análisis estadístico para buscar asociación entre variables, utilizando pruebas de Chi-cuadrado, test exacto de Fisher y regresión logística $(\mathrm{p}<0,05)$.

Resultados Encuesta respondida por 136 especialistas. Rango etario modal menor de 40 años (46,3\%), 94,1\% hombres. Un 53,7\% reportó burnout más de una vez al año, $11 \%$ anhedonia, 9,6\% ánimo depresivo más de la mitad del mes o casi todos los días y $59,6 \%$ cansancio diario. Se encontró asociación significativa entre burnout y edad menor de 40

\section{Palabras claves}

- burnout

- calidad de vida

- Chile

- medicina

- psicología

- traumatólogos años $(p=0,004)$, ausencia de hijos $(p=0,007)$, dieta no saludable $(p=0,001)$, anhedonia $(p=0,007)$, temor a demandas $(p<0,001)$ y cansancio $(p<0,003)$.

Conclusión Más de la mitad de los traumatólogos encuestados reportaron burnout más de una vez al año y cerca del $10 \%$ presentan síntomas depresivos semanalmente. Una edad menor a 40 años, anhedonia, el temor a demandas laborales y el cansancio al final de la jornada, fueron las variables de mayor impacto en la manifestación del burnout. La presencia de hijos y una dieta saludable fueron factores protectores.
\end{abstract}

received

July 20,2020

accepted

July 31,2020
DOI https://doi.org/

10.1055/s-0040-1719023.

ISSN 0716-4548.
Copyright $\odot 2020$ by Thieme Revinter

Publicações Ltda, Rio de Janeiro, Brazil
License terms

(c) $9 \ominus \$$ 
Abstract

\section{Keywords}

- burnout

- Chile

- medical

- orthopaedic surgeons

- psychological

- quality of life
Introduction There are no reports regarding the burnout index in Orthopaedic Surgeons in Chile.

Objective Assess the presence of burnout and other life quality aspects of this population.

Material and Methods Cross-Sectional analytical study. An originally designed survey was used to identify the self-report on burnout and depressive symptoms, amongst other factors. Demographic, work-related, social, personal habits, and mood-related variables were included. The survey was distributed by email to members of the Chilean Orthopaedic Surgery Society. Statistical analysis was conducted to determine the association between variables through the Chi-square test, Fisher's exact test, and logistic regressions $(\mathrm{p}<0.05)$.

Results The survey was answered by 136 orthopaedic surgeons. The modal age range was less than 40 years (46.3\%), and $94.1 \%$ were men. Of those surveyed, $53.7 \%$ reported a burnout episode more than once per year, $11 \%$ anhedonia, $9.6 \%$ a depressive mood more than half of the month or almost every day, and $59.6 \%$ of chronic fatigue. Statistical analysis showed a significant association between burnout and age under 40 years old $(p=0.004)$, not having children $(p=0.007)$, a non-healthy diet $(p=0.001)$, anhedonia $(p=0.007)$, fear of lawsuits $(p<0.001)$ and fatigue $(\mathrm{p}<0.003)$.

Conclusion More than half of the surveyed orthopaedic surgeons reported episodes of burnout more than once per year, and near $10 \%$ referred to have experienced weekly depressive symptoms. Age under 40 years, anhedonia, fear of lawsuits, and fatigue were the variables of more significant impact over the self-report of burnout. Having children and a healthy diet resulted in protective factors.

\section{Introducción}

El burnout es un término acuñado que hace referencia al desgaste o agotamiento laboral, haciendo su aparición durante el siglo XX bajo diferentes nombres como presión, estrés, sobrecarga o colapso laboral. ${ }^{1}$ Actualmente no es reconocido como patología, no obstante, es contemplado dentro de la Clasificación Internacional de Enfermedades (CIE-11) como un fenómeno ocupacional que afecta el estado de la salud. ${ }^{2}$ Más aún, hay elementos del burnout que se presentan como características comunes a otras patologías, tales como trastornos depresivos y del sueño. ${ }^{1,3}$

Ese fenómeno se halla en aumento a nivel mundial con tasas variadas dependiendo de la localidad, ocupación y herramienta utilizada para su medición. Se han encontrado altas tasas de burnout dentro del rubro médico, principalmente en especialidades quirúrgicas, explicadas por una mayor predisposición a rasgos compulsivos, sensación de culpa, negación y trabajar en una cultura laboral enfocada en el perfeccionismo, negación de la vulnerabilidad personal y posposición de la gratificación personal., ${ }^{4,5}$ En Estados Unidos se han descrito tasas cercanas al 50\% de burnout en cirujanos, con un incremento del $10 \%$ en los últimos años. ${ }^{1,6,7}$ Más preocupante aún, es que existen reportes con tasas de suicidio en cirujanos que serían más altas que las de otros trabajadores $^{1} \mathrm{y}$ estudios que han descrito una asociación directa entre burnout e ideación suicida. ${ }^{8-10}$
Los motivos que conducen al burnout en las especialidades quirúrgicas, son variados y cambian según la problemática que enfrentan los especialistas de cada región del globo. ${ }^{1}$ En China, uno de los principales factores de burnout es la carga laboral asociada a bajos sueldos; por otra parte, en Estados Unidos, sería el riesgo a las demandas médico-legales. ${ }^{1}$

Dentro de las especialidades quirúrgicas, los traumatólogos han sido considerados como un grupo de alto riesgo de burnout. ${ }^{5}$ Durante la última década en Chile, se ha observado una creciente carga asistencial en consultas y hospitalizaciones por ortopedia y traumatología, ${ }^{11}$ lo que puede favorecer a una sobrecarga laboral dentro de la especialidad y afectar no solo al tratante, sino también a la atención brindada a los pacientes. Pese a eso, no existen estudios nacionales de burnout enfocados en la especialidad de ortopedia y traumatología.

Este estudio tiene como objetivo evaluar la presencia de burnout y otros aspectos de la calidad de vida de los traumatólogos en Chile.

\section{Material y Método}

\section{Estudio transversal analítico}

Población y protocolo de estudio

A mediados del año 2019, previa autorización del comité de ética local, se distribuyó una encuesta voluntaria mediante 
un enlace dentro de una invitación por e-mail a 879 traumatólogos obtenidos de la base de datos de miembros de la Sociedad Chilena de Ortopedia y Traumatología (SCHOT) a lo largo del país. Esa encuesta, de carácter anónima, fue diseñada en formato Google Drive-Forms (Google LLC, Collaborative software Web survey, 2008).

La encuesta consistió en 29 preguntas de selección múltiple, dicotómicas y en escala de Likert (nivel de acuerdo con la afirmación). ${ }^{12}$ Se indagaron 4 secciones: demografía, laboral, social y personal (Archivo suplementario). Del total de preguntas, 2 de ellas (frecuencia de sensación de burnout e insensibilidad con terceros), son oficiales del cuestionario "Maslach Burnout Inventory" (MBI). ${ }^{13}$ Las preguntas fueron seleccionadas a través de un "focus group" formado por tres traumatólogos chilenos y una bioestadística, con el objetivo de crear una encuesta representativa local, inclusiva y fácil de contestar. La pregunta frecuencia de sensación de burnout, fue elegida como la variable principal del estudio para términos de análisis, al ser el elemento principal de la subescala "agotamiento emocional" en el cuestionario mencionado.

\section{Recolección de datos}

El plazo para recibir respuestas se cerró a las 2 semanas de enviada la encuesta, descargando posteriormente los resultados en una base de datos en formato Excel (Microsoft Excel®, Microsoft 365 MSO, 2019, Microsoft).

Análisis estadístico: Las respuestas fueron agrupadas en sub-categorías, a modo de facilitar el análisis de los datos.

Se obtuvieron frecuencias de las distintas variables y se buscó la asociación entre ellas y el burnout, mediante el Test exacto de Fisher para las variables no-paramétricas y Chicuadrado para las paramétricas. Posteriormente se realizó un análisis multivariado mediante regresión logística entre burnout y las variables más representativas de cada una de las cuatro secciones evaluadas, para medir su impacto como factor de riesgo o factor protector a través de sus Odds Ratio (OR). Se utilizó el programa estadístico SPSS ${ }^{\circledR}$ (IBM ${ }^{\circledR}$ Statistics for Windows, V. 24, 2016). Se estableció como significancia estadística un $p<0,05$.

\section{Ética}

Este estudio fue evaluado y aprobado por el comité de bioética de la Facultad Medicina Clínica Alemana Universidad del Desarrollo.

\section{Resultados}

De los 879 traumatólogos a quienes se les envió la encuesta, hubo una tasa de respuesta del $15 \%$, con una muestra final de 136 encuestados.

La asociación de burnout con los datos demográficos y laborales se resumen en la - Tabla 1, y con los elementos sociales y personales en la $\mathbf{- T a b l a ~ 2 . ~ S o ́ l o ~} 5$ variables no fueron incluidas en las tablas dada su cantidad de categorías y/o falta de significación estadística obtenida (casos operados, frecuencia de turnos, rol extracurricular, historia de demandas médico-legales y uso de medicamentos para conciliar el sueño).
Dentro de los resultados destacamos:

1) Demográfico: La mayoría de los encuestados correspondieron a hombres menores de 40 años de edad (46,3\%).

2) Laboral: Un 53,7\% reportó presentar episodios de burnout más de una vez al año y un 52,2\% manifestó sentirse insensible hacia sus pacientes con la misma frecuencia. El 49,3\% indicó sentir presión por temor a demandas médico-legales más de una vez al año.

3) Social: Más de un 75\% refirió estar casado y tener por lo menos 1 hijo.

4) Personal: Cerca del 10\% reportó anhedonia y/o ánimo depresivo más de la mitad del mes o casi todos los días. Aproximadamente un $60 \%$ indicó cansancio al final de la jornada laboral y el $80 \%$ satisfacción respecto de su carrera profesional.

Las variables que demostraron asociación significativa con burnout en el análisis bivariado, son edad joven (menor de 40 años) $(p=0,003)$, años ejercidos como especialista $(p=0,013)$, presión por temor a demandas médico-legales $(p<0,001)$, tener hijos $(p=0,006)$, dieta saludable $(p<0,001)$, cansancio al final de la jornada $(p=0,001)$, anhedonia $(p=0,011)$ y satisfacción por la carrera $(p<0,001)$. De esas variables, tener hijos y dieta saludable demostraron asociación significativa negativa, representado así factores protectores.

De esas variables, se escogieron las más representativas de cada sección para realizar una regresión logística, ilustrada en la - Tabla 3. Todas las variables demostraron asociación significativa en el análisis univariado, pero sólo el temor a demandas y cansancio al final de la jornada laboral, mantuvieron dicha asociación en el análisis multivariado.

\section{Discusión}

El burnout en los trabajadores de la salud se ha convertido en un tema de contingencia actual, dada la alta tasa reportada en estudios internacionales, especialmente en especialidades quirúrgicas. ${ }^{1,4-7}$ Ese fenómeno, además de afectar el bienestar personal del médico, puede impactar indirectamente en la calidad de atención de los pacientes. ${ }^{1}$ Pese a eso, se desconoce la presencia de burnout en traumatólogos dentro de Chile y Latinoamérica, ${ }^{14}$ por lo que el objetivo principal de este estudio fue evaluar el auto-reporte de dicho fenómeno en traumatólogos que ejercen en Chile.

Como muestra representativa para la realización de este estudio, se decidió difundir la encuesta a miembros de la SCHOT, dado que corresponde a una sociedad científica, que reúne cerca del $80 \%$ de los traumatólogos a lo largo del país.

Dentro de los resultados más significativos de este estudio, destaca que los traumatólogos más jóvenes $(<40$ años) fueron más propensos a reportar burnout que aquellos mayores ( $>60$ años), coincidiendo con publicaciones internacionales. $^{15}$ Eso podría explicarse porque en la actualidad, los especialistas más jóvenes presentan mayores expectativas sociales de balance trabajo-familia, 
Tabla 1 Elementos demográficos y laborales en asociación al burnout

\begin{tabular}{|c|c|c|c|c|}
\hline Variables & & $\begin{array}{l}\text { Sin burnout } \\
n=63(46 \%)\end{array}$ & $\begin{array}{l}\text { Con burnout } \\
\mathrm{n}=73(54 \%)\end{array}$ & Significancia estadística \\
\hline \multirow[t]{3}{*}{ Edad } & Adulto Joven (<40 años) & $22(16,1)$ & $41(30,1)$ & \multirow[t]{3}{*}{$p=0,003$} \\
\hline & Adulto (41-60 años) & $30(22)$ & $30(22)$ & \\
\hline & Adulto Mayor (>60 años) & $11(8)$ & $2(1,4)$ & \\
\hline \multirow[t]{2}{*}{ Sexo } & Masculino & $60(44,1)$ & $68(50)$ & \multirow[t]{2}{*}{$p=0,725$} \\
\hline & Femenino & $3(2,2)$ & $5(3,6)$ & \\
\hline \multirow[t]{5}{*}{ Años de especialidad } & $\leq 5$ años & $9(6,6)$ & $23(16,9)$ & \multirow[t]{5}{*}{$p=0,013$} \\
\hline & 6-10 años & $13(9,5)$ & $22(16,1)$ & \\
\hline & 11-20 años & $20(14,7)$ & $11(8)$ & \\
\hline & 21-30 años & $9(6,6)$ & $11(8)$ & \\
\hline & $>30$ años & $12(8,8)$ & $6(4,4)$ & \\
\hline \multirow[t]{4}{*}{ Horas de jornada laboral } & $\leq 7$ horas & $9(6,6)$ & $5(3,6)$ & \multirow[t]{4}{*}{$p=0,061$} \\
\hline & 8-9 horas & $17(12,5)$ & $27(19,8)$ & \\
\hline & 10-12 horas & $24(17,6)$ & $36(26,4)$ & \\
\hline & $>12$ horas & $12(8,8)$ & $5(3,6)$ & \\
\hline \multirow[t]{4}{*}{ Turnos en urgencia } & No & $44(32,3)$ & $42(30,8)$ & \multirow[t]{4}{*}{$p=0,068$} \\
\hline & Sí $<12$ horas & $4(2,9)$ & $4(2,9)$ & \\
\hline & Sí 12-24 horas & $6(4,4)$ & $20(14,7)$ & \\
\hline & Sí 24 horas & $9(6,6)$ & $7(5,1)$ & \\
\hline \multirow[t]{2}{*}{ Cargo administrativo } & Sí & $33(24,2)$ & $34(25)$ & \multirow[t]{2}{*}{$p=0,471$} \\
\hline & No & $30(22)$ & $39(28,6)$ & \\
\hline \multirow[t]{2}{*}{ Presión por temor a demandas } & Sí & $18(13,2)$ & $48(35,2)$ & \multirow[t]{2}{*}{$p<0,001$} \\
\hline & No & $45(33)$ & $24(17,6)$ & \\
\hline
\end{tabular}

así como de progreso y desarrollo personal y económico, mientras que los traumatólogos mayores podrían ya haber alcanzado un equilibrio económico y laboral. ${ }^{15}$ Otra posible causa podría ser el fenómeno conocido como "Fear of missing out" (miedo a perderse algo o "FOMO"), que se encuentra en ascenso en las poblaciones más jóvenes. ${ }^{16}$ Ese cuadro corresponde a la ansiedad que genera presenciar, a través de redes sociales, la experiencia que están viviendo terceros (amigos, familiares, conocidos y contactos), de las que no es posible participar directamente a consecuencia de otras responsabilidades. Eso puede llevar a la persona a sufrir angustia, desgaste emocional y cuestionamiento constante de su actividad laboral, ${ }^{16}$ pudiendo desencadenar un cuadro de burnout.

Respecto a la presencia de anhedonia y ánimo depresivo, aproximadamente 1 de cada 10 encuestados refirió sentir ambas más de la mitad de los días del mes. Las dos variables, corresponden a criterios diagnósticos de episodio depresivo. ${ }^{17}$ En el presente estudio, se encontró que la presencia de anhedonia fue directamente asociada con el reporte de burnout. Distintos autores han reportado una relación directa e independiente entre depresión y burnout. $^{8,18-21}$ Además, el riesgo de depresión entre las especialidades quirúrgicas también ha sido reportado, $^{1,8,22,23}$ lo cual podría repercutir en una baja calidad en la atención de los pacientes $\mathrm{y}$, asimismo, relacionarse con una mayor tasa de suicidio en esos especialistas. $^{8}$ Los datos encontrados deben ser reflexionados, pues se ha descrito que el auto-reporte de burnout se asocia significativamente con una mayor ideación suicida. ${ }^{8-10}$

La presión por temor a demandas, demostró la mayor asociación con el auto-reporte de burnout. En Chile, ha habido un incremento del número de procesos judiciales contra médicos especialistas en las últimas décadas, viendo a Traumatología como una de las 3 especialidades con más eventos. $^{24}$ Según Balch y col., el aumento de las acciones judiciales y de su difusión por parte de los medios y redes sociales, genera una angustia en el actuar y en la toma de decisiones del especialista, lo cual se asocia a un aumento en los errores cometidos por los médicos y a una mayor presencia de burnout. ${ }^{25}$

El presente estudio mostró una tendencia estadística, en que las mujeres presentaron un mayor reporte de burnout que los hombres. El hecho que no se lograra significancia, posiblemente se debió al número reducido de mujeres encuestadas (5,8\% del universo), lo cual coincide con el porcentaje de especialistas de sexo femenino, miembros de 
80 Burnout y calidad de vida en traumatólogos chilenos Vaisman y col.

Tabla 2 Elementos sociales y personales en asociación al burnout

\begin{tabular}{|c|c|c|c|c|}
\hline Variables & & $\begin{array}{l}\text { Sin burnout } \\
n=63(46 \%)\end{array}$ & $\begin{array}{l}\text { Con burnout } \\
\mathrm{n}=73(54 \%)\end{array}$ & Significancia estadística \\
\hline \multirow[t]{3}{*}{ Estado civil } & Casado(a) & $48(35,2)$ & $55(40,4)$ & \multirow[t]{3}{*}{$p=0,481$} \\
\hline & Soltero(a) & $6(4,4)$ & $11(8)$ & \\
\hline & Divorciado(a) & $9(6,6)$ & $7(5,1)$ & \\
\hline \multirow[t]{2}{*}{ Hijos } & Con hijos & $55(40,4)$ & $49(36)$ & \multirow[t]{2}{*}{$p=0,006$} \\
\hline & Sin Hijos & $8(5,8)$ & $24(17,6)$ & \\
\hline \multirow[t]{5}{*}{ Consumo de alcohol } & No & $8(5,8)$ & $5(3,6)$ & \multirow[t]{5}{*}{$p=0,624$} \\
\hline & $\leq 1 \mathrm{vez}$ al mes & $11(8)$ & $12(8,8)$ & \\
\hline & $2-4$ veces al mes & $21(15,4)$ & $32(23,5)$ & \\
\hline & $2-3$ veces a la semana & $17(12,5)$ & $16(11,7)$ & \\
\hline & $\geq 4$ veces a la semana & $6(4,4)$ & $8(5,8)$ & \\
\hline \multirow[t]{2}{*}{ Consumo de drogas } & Sí & $5(3,6)$ & $5(3,6)$ & \multirow[t]{2}{*}{$p=1,0$} \\
\hline & No & $58(42,6)$ & $68(50)$ & \\
\hline \multirow[t]{2}{*}{ Dieta } & Saludable & $42(30,8)$ & $28(20,5)$ & \multirow[t]{2}{*}{$P<0,001$} \\
\hline & No saludable & $4(2,9)$ & $29(21,3)$ & \\
\hline \multirow[t]{2}{*}{ Horas de sueño } & $\leq 6$ horas & $35(25,7)$ & $48(35,2)$ & \multirow[t]{2}{*}{$p=0,152$} \\
\hline & $7-8$ horas & $28(20,5)$ & $23(16,9)$ & \\
\hline \multirow[t]{2}{*}{ Cansancio al final de la jornada } & Sí & $28(20,5)$ & $53(38,9)$ & \multirow[t]{2}{*}{$p=0,001$} \\
\hline & No & $34(25)$ & $20(14,7)$ & \\
\hline \multirow[t]{2}{*}{ Anhedonia } & Sí & $2(1,4)$ & $13(9,5)$ & \multirow[t]{2}{*}{$p=0,011$} \\
\hline & No & $61(44,8)$ & $60(44,1)$ & \\
\hline \multirow[t]{2}{*}{ Ánimo depresivo } & Sí & $3(2,2)$ & $10(7,3)$ & \multirow[t]{2}{*}{$p=0,088$} \\
\hline & No & $60(44,1)$ & $63(46,3)$ & \\
\hline \multirow[t]{2}{*}{ Satisfacción por la carrera } & Sí & $59(43,3)$ & $50(36,7)$ & \multirow[t]{2}{*}{$p<0,001$} \\
\hline & No & $4(2,9)$ & $23(16,9)$ & \\
\hline
\end{tabular}

Tabla 3 Regresión Logística de variables representativas con burnout

\begin{tabular}{|c|c|c|c|c|c|c|}
\hline \multirow[t]{2}{*}{ Variables } & \multirow[t]{2}{*}{$\mathrm{N}^{\circ}$ Casos } & \multirow{2}{*}{$\begin{array}{l}\text { Casos con } \\
\text { burnout }\end{array}$} & \multicolumn{2}{|l|}{ Análisis Univariado } & \multicolumn{2}{|l|}{ Análisis Multivariado } \\
\hline & & & $\begin{array}{l}\text { Odds Ratio } \\
\text { No ajustado (IC 95\%) }\end{array}$ & Valor $p$ & $\begin{array}{l}\text { Odds Ratio } \\
\text { Ajustado (IC 95\%) }\end{array}$ & Valor $p$ \\
\hline Adulto Mayor (>60 años) & 13 & 2 & $1^{*}$ & - & $1^{*}$ & - \\
\hline Adulto (41-60 años) & 60 & 30 & $5,5(1,22-27)$ & 0,036 & $4(0,658-24,3)$ & 0,249 \\
\hline Adulto Joven ( $<40$ años) & 61 & 41 & $10,5(2,12-51,7)$ & 0,004 & $3,01(0,482-19,7)$ & 0,321 \\
\hline Presión por temor a demandas & 67 & 49 & $5,1(2,45-10,6)$ & $<0,001$ & $3,58(1,33-9,66)$ & 0,012 \\
\hline Tener Hijos & 104 & 49 & $0,3(0,122-0,722)$ & 0,007 & $0,366(0,094-1,42)$ & 0,146 \\
\hline Dieta Saludable & 70 & 28 & $0,388(0,220-0,684)$ & 0,001 & $0,286(0,075-1,09)$ & 0,067 \\
\hline Cansancio al final de la jornada & 128 & 73 & $3,3(1,73-6,29)$ & $<0,001$ & $2,73(1,02-7,31)$ & 0,045 \\
\hline
\end{tabular}

*Referencia para comparaciones; IC: Intervalo de confianza.

la SCHOT (6,7\%) (Iñiguez M, y col., "Mujeres en Traumatología en Chile”, observaciones no publicadas). Ese porcentaje de mujeres especialistas en Ortopedia y Traumatología, coincide con otros reportes internacionales ${ }^{26,27} \mathrm{y}$ autores como Archer y McCue, han asociado al sexo femenino como un factor de riesgo significativo para la presencia de estrés emocional en Ortopedistas. ${ }^{28,29}$

Si bien la cantidad de horas de sueño no alcanzó una asociación significativa, esta sí muestra una tendencia inversa, donde quienes duermen 6 horas o menos exhiben 
aproximadamente un $20 \%$ más de burnout respecto de quienes duermen 7 a 8 horas. Probablemente, frente a un mayor " $n$ " muestral, esa tendencia se haría significativa. Múltiples publicaciones han descrito la asociación entre las horas de sueño y el burnout, indicando una relación inversa entre ambos, además de múltiples efectos adversos, tanto en la salud física como mental. ${ }^{30,31}$

En lo que respecta al contexto laboral, se observó una asociación significativa entre el cansancio al final de la jornada y el auto-reporte de burnout. Particularmente, realizar turnos de urgencia y un mayor número de horas en la jornada laboral, no se asociaron significativamente con una mayor tendencia al burnout. Sin embargo, destaca una tendencia a mayor reporte de burnout en aquellos profesionales que trabajan en jornadas diarias de 8 a 12 horas, al igual que quienes hacen turnos de urgencia de 12 a 24 horas por semana. Al respecto, Shanafelt y col., demostraron una asociación significativa entre turnos de urgencia nocturnos, un alto número de horas de jornada laboral y burnout, respaldando la tendencia observada en este estudio., 5, Tener una sobrecarga laboral deja menos tiempo para realizar actividades potencialmente protectoras de burnout como son: dormir un número adecuado de horas, compartir con familia y amistades, tener tiempo para hobbies y ejercicios físicos, trabajar el aspecto espiritual y tomar vacaciones. ${ }^{5}$

En el presente estudio, tener hijos y mantener una dieta saludable, destacaron como factores protectores. Pese a que existe controversia al respecto, múltiples autores coinciden en que tener hijos disminuye la presencia de burnout, planteando a la familia como un pilar de apoyo emocional para discutir, desviar o enfrentar el estrés laboral o como una motivación para sobrellevarlo. ${ }^{5,7,33}$ Una dieta saludable es un factor protector claramente avalado en la literatura. Además de disminuir el reporte de estrés y de síntomas depresivos, otorga múltiples beneficios sobre la salud física y el bienestar general del individuo. ${ }^{34}$

Este estudio destaca por ser el primer trabajo que analiza el auto-reporte de burnout en traumatólogos que ejercen en Chile. Sin embargo, no se encuentra exento de limitaciones. En primer lugar, una tasa de respuesta del $15 \%$ puede representar un sesgo de selección, donde la población más afectada por burnout y por estrés laboral, sintió la mayor motivación por responder la encuesta, generando así una posible sobreestimación del evento a medir. No obstante, ese es un sesgo presente en todas las encuestas voluntarias, obteniendo tasas sumamente variables (6\%-70\%) dependiendo de elementos como el método a través del cual se realizó la encuesta o el uso de incentivos, ${ }^{35}$ y otros factores así como la longitud de la encuesta y el formato de las preguntas. $^{36}$ En segundo lugar, cabe cuestionarse la validez de esta encuesta respecto del diagnóstico de burnout dado que, para fines prácticos, no se integró completamente el cuestionario $\mathrm{MBI}$, ni tampoco se consideró la situación económica y financiera de los encuestados. Sin embargo, valoramos la simplificación de la evaluación del burnout, dando espacio a analizar otras variables significativas para la realidad nacional. El incluir el cuestionario MBI completo habría prolongado el tiempo requerido para contestar la encuesta, con una potencial reducción en la tasa de respuestas. Pese a ese potencial sesgo, los resultados del presente estudio son, en su mayoría, comparables con la literatura internacional. 5,7,30,31,33,34 Finalmente, este reporte fue realizado sólo en especialistas en Ortopedia y Traumatología, por lo que no necesariamente refleja la realidad de otras especialidades. Sin embargo, existen reportes internacionales similares entre especialidades quirúrgicas ${ }^{1,5,8,22,23} \mathrm{y}$ en médicos en formación en Chile. ${ }^{37,38}$ De todas formas, es recomendable explorar el reporte de burnout en otras especialidades médicoquirúrgicas, utilizando esa misma metodología.

A partir de los hallazgos del presente estudio, se identificaron variables modificables que podrían reducir la incidencia de burnout y mejorar la percepción de calidad de vida del Traumatólogo. Se recomienda: integrar a la vida diaria una dieta saludable, promover el apoyo familiar y conciliar 7-8 horas de sueño. Esas simples medidas están respaldadas en la literatura. ${ }^{5,7,30,31,33,34}$

Como conclusión, más de la mitad de los traumatólogos encuestados reportaron burnout más de una vez al año y cerca del $10 \%$ presentan síntomas depresivos semanalmente. Una edad menor a 40 años, anhedonia, el temor a demandas laborales y el cansancio al final de la jornada, fueron las variables de mayor impacto en la manifestación del burnout. La presencia de hijos y una dieta saludable fueron factores protectores.

\section{Conflictos de intereses}

El Dr. D. Figueroa reporta ser consultor pagado de Stryker, Smith and Nephew y Conmed, no obstante, esto no guarda relación con el trabajo el presente trabajo publicado. Es director de la of Revista Chilena de Ortopedia y Traumatología, miembro de la junta editorial del Journal of ISAKOS, consultor de la Revista de Artroscopía y Secretario de la junta de Directores de ISAKOS. El Dr. F. Figueroa reporta ser Editor del Acta Ortopédica Mexicana. El Dr. Calvo reporta ser consultor pagado de Stryker y Smith and Nephew, no obstante, esto no guarda relación con el trabajo el presente trabajo publicado. Es Editor de la Revista Chilena de Ortopedia y Traumatología. El Dr. Vaisman reporta ser consultor pagado de Arthrex, no obstante, esto no guarda relación con el trabajo el presente trabajo publicado. Es Editor de la Revista Chilena de Ortopedia y Traumatología y Orthopaedic Journal of Sports Medicine. Además, es Tesorero de la junta de Directores de la Sociedad Chilena de Ortopedia y Traumatología.

\section{Referencias}

1 van Dijk CN. Are we surgeons finding it all too much? Dealing with the pressures of our profession.J ISAKOS Jt Disord Orthop Sport Med 2018;3(03):125-127. Doi: 10.1136/jisakos-2018-000237

2 World Health Organization. Burn-out an "occupational phenomenon": International Classification of Diseases. https://www.who.int/ mental_health/evidence/burn-out/en/. Accessed July 7, 2020

3 Masluk B. Dificultades Y Comorbilidad En El Diagnóstico De Burnout. El Aspecto Legal De Su Reconocimiento. XVII Congr Virtual Int Psiquiatr. 2016;(February):9. https://www. 
researchgate.net/publication/303885011_DIFICULTADES_Y_ COMORBILIDAD_EN_EL_DIAGNOSTICO_DE_BURNOUT_EL_ ASPECTO_LEGAL_DE_SU_RECONOCIMIENTO

4 Gazelle G, Liebschutz JM, Riess H. Physician burnout: coaching a way out. J Gen Intern Med 2015;30(04):508-513. Doi: 10.1007/ s11606-014-3144-y

5 Shanafelt TD, Balch CM, Bechamps GJ, et al. Burnout and career satisfaction among American surgeons. Ann Surg 2009;250(03): 463-471. Doi: 10.1097/SLA.0b013e3181ac4dfd

6 Dyrbye LN, Shanafelt TD, Balch CM, Satele D, Sloan J, Freischlag J. Relationship between work-home conflicts and burnout among American surgeons: a comparison by sex. Arch Surg 2011;146 (02):211-217. Doi: 10.1001/archsurg.2010.310

7 Dimou FM, Eckelbarger D, Riall TS. Surgeon burnout: A systematic review. J Am Coll Surg 2016;222(06):1230-1239. Doi: 10.1016/j. jamcollsurg.2016.03.022

8 Shanafelt TD, Balch CM, Dyrbye L, et al. Special report: suicidal ideation among American surgeons. Arch Surg 2011;146(01): 54-62. Doi: 10.1001/archsurg.2010.292

9 van der Heijden F, Dillingh G, Bakker A, Prins J. Suicidal thoughts among medical residents with burnout. Arch Suicide Res 2008;12 (04):344-346. Doi: 10.1080/13811110802325349

10 Dyrbye LN, Thomas MR, Massie FS, et al. Burnout and suicidal ideation among U.S. medical students. Ann Intern Med 2008;149 (05):334-341. Doi: 10.7326/0003-4819-149-5-200809020-00008

11 Salud D de E e I de. Egresos Hospitalarios. http://www.deis.cl/ bases-de-datos-egresos-hospitalarios/. Published 2018

12 Likert R. A Technique for the Measurement of Attitudes. Arch Phychology 1932. Doi: 10.4135/9781412961288.n454

13 Maslach C, Jackson SE. The measurement of experienced burnout. J Organ Behav 1981;2(02):99-113. Doi: 10.1002/job.4030020205

14 Astudillo MP, Losada MH, Schneeberger HP, Coronado F, Curitol SS. Prevalencia de Síndrome de Burnout en un Centro de Cirugía Académico-Asistencial Público en Chile. Rev Chil Cir 2018;70(02): 117-126. Doi: 10.4067/s0718-40262018000200117

15 Campbell DA Jr, Sonnad SS, Eckhauser FE, Campbell KK, Greenfield LJ. Burnout among American surgeons. Surgery 2001;130(04): 696-702, discussion 702-705. Doi: 10.1067/msy.2001.116676

16 FOMO. It's your life you're missing out on - ScienceDaily. https:// www.sciencedaily.com/releases/2016/03/160330135623.htm. Accessed July 7, 2020

17 Ministerio de Salud de Chile. Guía Clínica Depresión en personas de 15 años y más. Ser Guías cínicas Minsal 2013https://www. minsal.cl/portal/url/item/ 7222754637c08646e04001011f014e64.pdf

18 Shanafelt TD, Gorringe G, Menaker R, et al. Impact of organizational leadership on physician burnout and satisfaction. Mayo Clin Proc 2015;90(04):432-440. Doi: 10.1016/j.mayocp.2015.01.012

19 Bianchi R, Schonfeld IS, Laurent E. Burnout-depression overlap: a review. Clin Psychol Rev 2015;36:28-41. Doi: 10.1016/j. cpr.2015.01.004

20 Asai M, Morita T, Akechi T, et al. Burnout and psychiatric morbidity among physicians engaged in end-of-life care for cancer patients: a cross-sectional nationwide survey in Japan. Psychooncology 2007;16(05):421-428. Doi: 10.1002/pon.1066

21 Patel RS, Bachu R, Adikey A, Malik M, Shah M. Factors Related to Physician Burnout and Its Consequences: A Review. Behav Sci (Basel) 2018;8(11):98. Doi: 10.3390/bs8110098

22 Balch CM, Shanafelt TD, Sloan J, Satele DV, Kuerer HM. Burnout and career satisfaction among surgical oncologists compared with other surgical specialties. Ann Surg Oncol 2011;18(01): 16-25. Doi: 10.1245/s10434-010-1369-5
23 Goldstein RY, Weiss JM. The Canary in the Coal Mine: Wellness Among Pediatric Orthopedic Surgeons. Orthop Clin North Am 2019;50(03):327-330. Doi: 10.1016/j.ocl.2019.03.009

24 Fundación de Asistencia Legal de Colegio Médico. Cuenta Pública 2017. https://www.falmed.cl/Portals/0/files/ 181119cuenta_publica2017.pdf. Accessed July 7, 2020

25 Balch CM, Oreskovich MR, Dyrbye LN, et al. Personal consequences of malpractice lawsuits on American surgeons. J Am Coll Surg 2011;213(05):657-667. Doi: 10.1016/j.jamcollsurg.2011.08.005

26 Chambers CC, Ihnow SB, Monroe EJ, Suleiman LI. Women in Orthopaedic Surgery: Population Trends in Trainees and Practicing Surgeons. J Bone Joint Surg Am 2018;100(17):e116. Doi: $10.2106 / J B J S .17 .01291$

27 Poon S, Kiridly D, Mutawakkil M, et al. Current Trends in Sex, Race, and Ethnic Diversity in Orthopaedic Surgery Residency. J Am Acad Orthop Surg 2019;27(16):e725-e733. Doi: 10.5435/JAAOS-D-1800131

28 Archer LR, Keever RR, Gordon RA, Archer RP. The relationship between residents' characteristics, their stress experiences, and their psychosocial adjustment at one medical school. Acad Med 1991;66(05):301-303. Doi: 10.1097/00001888-19910500000018

29 McCue JD. The distress of internship. Causes and prevention. N Engl J Med 1985;312(07):449-452. Doi: 10.1056/ NEJM198502143120725

30 Lin YL, Chen CH, Chu WM, et al. Modifiable risk factors related to burnout levels in the medical workplace in Taiwan: crosssectional study. BMJ Open 2019;9(11):e032779. Doi: 10.1136/ bmjopen-2019-032779

31 Stewart NH, Arora VM. The Impact of Sleep and Circadian Disorders on Physician Burnout. Chest 2019;156(05): 1022-1030. Doi: 10.1016/j.chest.2019.07.008

32 Shanafelt TD, Oreskovich MR, Dyrbye LN, et al. Avoiding burnout: the personal health habits and wellness practices of US surgeons. Ann Surg 2012;255(04):625-633. Doi: 10.1097/ SLA.0b013e31824b2fa0

33 Sargent MC, Sotile W, Sotile MO, Rubash H, Barrack RL. Stress and coping among orthopaedic surgery residents and faculty. J Bone Joint Surg Am 2004;86(07):1579-1586. Doi: 10.2106/00004623200407000-00032

34 Hamidi MS, Boggild MK, Cheung AM. Running on empty: a review of nutrition and physicians' well-being. Postgrad Med J 2016;92(1090):478-481. Doi: 10.1136/postgradmedj-2016134131

35 Brtnikova M, Crane LA, Allison MA, Hurley LP, Beaty BL, Kempe A. A method for achieving high response rates in national surveys of U.S. primary care physicians. PLoS One 2018;13(08):e0202755. Doi: 10.1371/journal.pone.0202755

36 Hardigan PC, Popovici I, Carvajal MJ. Response rate, response time, and economic costs of survey research: A randomized trial of practicing pharmacists. Res Social Adm Pharm 2016;12(01): 141-148. Doi: 10.1016/j.sapharm.2015.07.003

37 Bitran M, Zúñiga D, Pedrals N, et al. Burnout en la formación de profesionales de la salud en Chile: Factores de protección y riesgo, y propuestas de abordaje desde la perspectiva de los educadores. Rev Med Chil 2019;147(04):510-517. Doi: 10.4067/s003498872019000400510

38 Díaz Piga LA, Arab Verdugo JP, Nuñez Palma C, et al. Burnout en médicos residentes de especialidades y subespecialidades: estudio de prevalencia y variables asociadas en un centro universitario. ARS MEDICA Rev Ciencias Médicas 2017;42(02): 27-33. Doi: 10.11565/arsmed.v42i2.541 